\title{
Valeurs et rapport texte/image dans l'album de littérature de jeunesse : étude d'un exemple, Le Génie du pousse-pousse
}

Values and Image-Text Relationship in Children's Literature: Analysis of an Example, Le Génie du pousse-pousse

\section{Anne Leclaire-Halté}

\section{(2) OpenEdition}

\section{Journals}

Édition électronique

URL : http://journals.openedition.org/pratiques/2259

DOI : $10.4000 /$ pratiques.2259

ISSN : 2425-2042

Éditeur

Centre de recherche sur les médiations (CREM)

Édition imprimée

Date de publication : 31 décembre 2014

Référence électronique

Anne Leclaire-Halté, « Valeurs et rapport texte/image dans l'album de littérature de jeunesse : étude d'un exemple, Le Génie du pousse-pousse », Pratiques [En ligne], 163-164 | 2014, mis en ligne le 31 décembre 2014, consulté le 19 avril 2019. URL : http://journals.openedition.org/pratiques/2259 ; DOI : $10.4000 /$ pratiques.2259

Ce document a été généré automatiquement le 19 avril 2019

(c) Tous droits réservés 


\title{
Valeurs et rapport texte/image dans l'album de littérature de jeunesse : étude d'un exemple, Le Génie du pousse-pousse
}

\author{
Values and Image-Text Relationship in Children's Literature: Analysis of an \\ Example, Le Génie du pousse-pousse
}

Anne Leclaire-Halté

1 Tout texte, tout ouvrage est porteur de valeurs, et la littérature de jeunesse n'échappe pas à ce constat. Pour l'enseignement à l'école primaire, dans les écrits à visée didactique, la relation entre littérature de jeunesse et valeurs ${ }^{1}$ est abordée suivant deux axes principaux.

2 Le premier est celui de la discussion à visée philosophique (DVP), champ bien exploré ces 20 dernières années. La DVP est issue des travaux de M. Lipman, philosophe américain pionnier dans la pratique de la philosophie à l'école. Certains auteurs, qui situent leur réflexion dans cet axe, sans toutefois prôner le protocole exact proposé par M. Lipman pour la DVP, reconnaissent la littérature de jeunesse comme un matériau intéressant pour faire éclore des problématiques de discussion liées aux valeurs et aux questions morales (voir par exemple, Leleux, 2009; Chirouter, 2007, 2012, 2014). E. Chirouter, par exemple (2014), considère la littérature comme une intéressante médiation pour amener les élèves à penser, dans le cadre d'une activité philosophique.

3 Le second axe est celui du débat interprétatif. Ce qu'A. Dias-Chiaruttini (2007) considère comme un genre disciplinaire émerge à la fin des années 90 et apparait dans les programmes de l'école primaire de 2002. Le débat interprétatif, tel qu'il est évoqué en didactique de la littérature, a été divulgué surtout grâce aux travaux de C. Tauveron (par exemple, 2002, 2004). Le terme recouvre, dans les classes, des réalités diversifiées, mais, pour $\mathrm{C}$. Tauveron, il renvoie à une pratique à distinguer du débat réflexif (encore que la frontière entre débat réflexif, plutôt associé aux pratiques philosophiques, et débat interprétatif semble parfois ténue). Le débat interprétatif ne fait pas de l'œuvre étudiée 
un prétexte à faire parler les élèves sur telle ou telle question (ce qui, pour C. Tauveron, relève plutôt du débat réflexif) et refuse l'entrée thématique. Il renvoie à une approche littéraire des textes, destinée à repérer et à interroger les «blancs » de ces derniers, qui sont à choisir par l'enseignant parce qu'ils sont réticents. Le débat interprétatif peut conduire à discuter de questions morales, si on considère la morale comme l'« ensemble des normes ou règles de conduite admises dans un domaine d'activité particulier, dans un groupe social particulier à une époque donnée » (selon la définition donnée par le Trésor de la langue française informatisé), si la morale est définie, dans le contexte qui est le nôtre, comme l'ensemble des valeurs préconisées par l'institution scolaire car supposées être les meilleures pour le sujet tant individuellement que socialement. Mais le débat interprétatif n'a pas, en soi, de visée morale.

Si la visée morale consiste à préconiser les valeurs qui sont bonnes pour le sujet, pris individuellement ou dans sa vie sociale, nous situons notre contribution à ce numéro un peu en aval de cette question. Se positionner par rapport aux valeurs présentes dans une œuvre de littérature de jeunesse, quelle qu'elle soit, nous semble une compétence fondamentale de lecture à développer chez les élèves, et ce, dès l'école primaire. Et, pour se situer par rapport à ces valeurs, il est nécessaire d'accéder à l'orientation axiologique qu'imprime à cette œuvre son auteur. Nous ne défendons pas l'idée d'une approche techniciste par les élèves, ni celle d'une étude en règle des dispositifs textuels mis en œuvre, consciemment ou non, par l'auteur. Mais il nous semble important que l'enseignant guide les élèves dans cette lecture des valeurs, et ce guidage sera d'autant plus effectif si, au préalable, un repérage des dispositifs sémiotiques présents dans l'ouvrage abordé en classe a été opéré. Comme le note A. Petitjean (2014),

« toute lecture est nécessairement subjective dans la mesure où tout texte de fiction est à des degrés divers indéterminé et narcotisé et que chaque lecteur projette dans le texte sa mémoire personnelle et les valeurs incorporées à sa socio-culture. Il n'en demeure pas moins qu'il existe une perspective axiologique de l'instance énonciative et qu'il est opportun de faire la part entre les valeurs objectivables à partir des données textuelles et les valeurs subjectives investies par le lecteur ».

5 À l'école primaire, l'album est la forme qui est souvent choisie par l'enseignant pour ses élèves. C'est pourquoi nous nous intéresserons, dans le cadre de cet article, à la façon dont texte et image interagissent dans l'orientation axiologique de l'album, ce qui, à notre connaissance, n'a encore fait l'objet d'aucune étude.

6 Précisons d'emblée que cet article, contrairement à d'autres dans ce dossier, ne traite pas de questions liées à la philosophie morale ou à son épistémologie, mais de l'inscription des valeurs dans les albums, en mettant en exergue l'importance de la relation texteimage dans cette inscription, ce qui nous semble insuffisamment développé, à l'heure où, pourtant, la maitrise des supports pluricodés, de plus en plus prégnants et aussi différenciateurs ${ }^{2}$ aujourd'hui, pourrait être initiée dès l'école primaire.

7 Après un rappel de quelques notions auxquelles nous aurons recours dans notre analyse, nous étudierons un album, Le Génie du pousse-pousse, écrit par J.-C. Noguès et illustré par A. Roby. Cet ouvrage a été publié par les éditions Milan pour la première fois en 2001, et a été réédité plusieurs fois depuis. Il ne s'agit pas d'un iconotexte au sens strict, ce terme 
étant plutôt réservé aux albums où auteur et illustrateur se confondent, mais d'un album illustré (Van der Linden, 2008).

Pourquoi un tel choix ? Plusieurs raisons peuvent être avancées :

- l'ouvrage figure sur le site Éduscol ${ }^{3}$, dans la sélection proposée dans la liste de référence de 2013 pour le cycle 3 ;

- il est l'objet d'un certain nombre de propositions d'exploitation pédagogique, à destination des enseignants, sur l'internet ;

- il relève d'un genre, le conte philosophique oriental, dont une des caractéristiques est de délivrer un message de sagesse ; on peut donc supposer qu'il s'agira d'un album qui rendra plus aisé l'accès à des dispositifs générateurs de valeurs.

\section{Quelques éléments de cadrage}

\subsection{Qu'entendre par valeurs?}

J.-M. Klinkenberg (2011) souligne l'extrême labilité du terme valeur. Il est vrai que ce dernier peut présenter diverses significations. La valeur peut être le caractère mesurable d'un objet susceptible d'être échangé, désiré : on parle de la valeur d'un terrain, d'un objet de valeur, des valeurs boursières. Le mot peut renvoyer aussi à une évaluation positive que l'on porte sur un objet, jugé estimable selon les normes d'un groupe social défini ; il est alors synonyme de qualité. Il désigne également les normes elles-mêmes, en tant qu'éléments de référence dans la conduite et les jugements : il est question des valeurs d'une société, d'un parti politique, de leur système de valeurs. Dans certains domaines, le terme est utilisé pour parler d'une mesure, par exemple en musique celle de la durée des notes et des silences, ou en peinture celle des degrés d'intensité dans les couleurs.

Selon les champs théoriques (philosophie, logique, économie, sociologie, psychologie, etc.), les valeurs sont ainsi définies et classées de manière différente. Et même au sein d'un même champ, les typologies varient selon les théoriciens, les objectifs poursuivis par la description.

11 Pour notre part, nous nous réfèrerons aux travaux d'A. J. Greimas, $(1983,1993)$ qui montrent comment s'effectue le passage de la valeur prise au sens saussurien, c'est-à-dire comme sens constitué par la mise en relation d'un signe avec d'autres signes du même système, à la valeur dans son acception axiologique. Linguistiquement, A. J. Greimas la définit d'abord comme un sème, une virtualité parmi d'autres servant à constituer un lexème. Seule la manifestation discursive permet de préciser les virtualités retenues, c'est-à-dire les valeurs du lexème, qui se fixent dans le point syntaxique qu'est l'objet. La valeur n'est donc perceptible en tant que telle qu'une fois inscrite dans la structure syntaxique, et la valeur investie dans l'objet devient une valeur pour le sujet à partir du moment où celui-ci vise l'objet. A. J. Greimas (1983 : 23) note :

«Dans la mesure où l'énoncé élémentaire peut se définir comme relation orientée engendrant ses deux termes-aboutissants - le sujet et l'objet-, la valeur qui s'investit dans l'objet visé sémantise en quelque sorte l'énoncé tout entier et devient du coup la valeur du sujet qui la rencontre en visant l'objet, et le sujet se trouve déterminé dans son existence sémantique par sa relation à la valeur. Il suffira donc, dans une étape ultérieure, de doter le sujet d'un vouloir-être pour que la valeur du sujet, au sens sémiotique, se change en valeur pour le sujet, au sens axiologique de ce terme. » 
12 Nous nous inspirons de cette réflexion pour définir la valeur comme une préférence qu'un sujet marque pour un objet; nous réservons donc ce terme à tout ce qui est axiologisé positivement par le sujet (on pourra appeler anti-valeur ce que celui-ci axiologise négativement. Tout peut être objet de préférence et il n'y a pas de valeur en soi, comme le remarque G. Genette (199: 63) :

« On peut, me semble-t-il, poser en principe qu'aucune valeur d'aucune sorte n'est objective et absolue, parce que rien, par définition, ne peut présenter de "valeur", c'est-à-dire valoir quelque chose, qu'aux yeux de quelqu'un ou de quelques-uns : valoir, c'est inévitablement valoir-pour : toute valeur est, en ce sens, relative. Tel objet vaut beaucoup pour tel sujet, beaucoup moins, voire rien du tout pour tel autre. Il peut advenir, par hasard ou par nécessité, et j'y reviendrai, qu'il vaille autant pour tous, mais il ne se peut qu'il vaille (quoi que ce soit) en lui-même, indépendamment d'un ou plusieurs sujets qui l'évaluent - ou le valorisent. "

Cette conception de la valeur n'intègre pas la dimension morale, puisque la question de savoir si ce qui est valeur pour le sujet est désirable dans la perspective d'une vie bonne ne se pose pas.

Objet et valeur, dans nos propos antérieurs, tendent à s'assimiler. Or, ils peuvent être dissociés, ce que A. J. Greimas (1984 : 21) explique avec l'exemple suivant :

«Lorsque quelqu'un, par exemple, se porte acquéreur, dans notre société d'aujourd'hui, d'une voiture automobile, ce n'est peut-être pas tellement la voiture en tant qu'objet qu'il veut acquérir, mais d'abord un moyen de déplacement rapide, substitut moderne du tapis volant d'autrefois; ce qu'il achète souvent, c'est aussi un peu de prestige social ou un sentiment de puissance plus intime. L'objet visé n'est alors qu'un prétexte, qu'un lieu d'investissement des valeurs, un ailleurs qui médiatise le rapport du sujet à lui-même. »

15 L'objet figuratif visé est dans ce cas à distinguer des valeurs elles-mêmes, qui sont à inférer de cet objet figuratif.

\subsection{La notion de scripteur}

16 Nous aurons recours, dans l'analyse qui suit, au terme scripteur. J. Peytard (1982:140), dans un schéma dressant la topographie des instances du champ littéraire, en distingue trois qu'il appelle situationnelle, ergo-textuelle et textuelle. Du côté de la production, l'instance situationnelle est l'auteur, en tant qu'être défini par un nom, une biographie, un ancrage dans un contexte sociopolitique. Toujours en production, l'instance ergotextuelle est le scripteur, c'est-à-dire le sujet qui commet l'acte d'écrire, qui construit le texte par un travail sur le langage. Le scripteur préside à l'orientation axiologique globale du texte, en manipulant les instances textuelles que sont le narrateur et les personnages, sujets intra-textuels susceptibles eux-mêmes de manifester leurs préférences, explicitement ou de façon plus indirecte.

17 Déterminer les valeurs du narrateur et des personnages et étudier leur hiérarchisation aide à repérer celles du scripteur. Précisons à ce propos que si ce dernier constitue une instanciation de l'auteur, il est en même temps une image construite par le lecteur, qui n'a pas d'autre choix que de "remonter » jusqu'à lui à partir du narrateur et des personnages. 


\section{Analyse du texte de l'album}

18

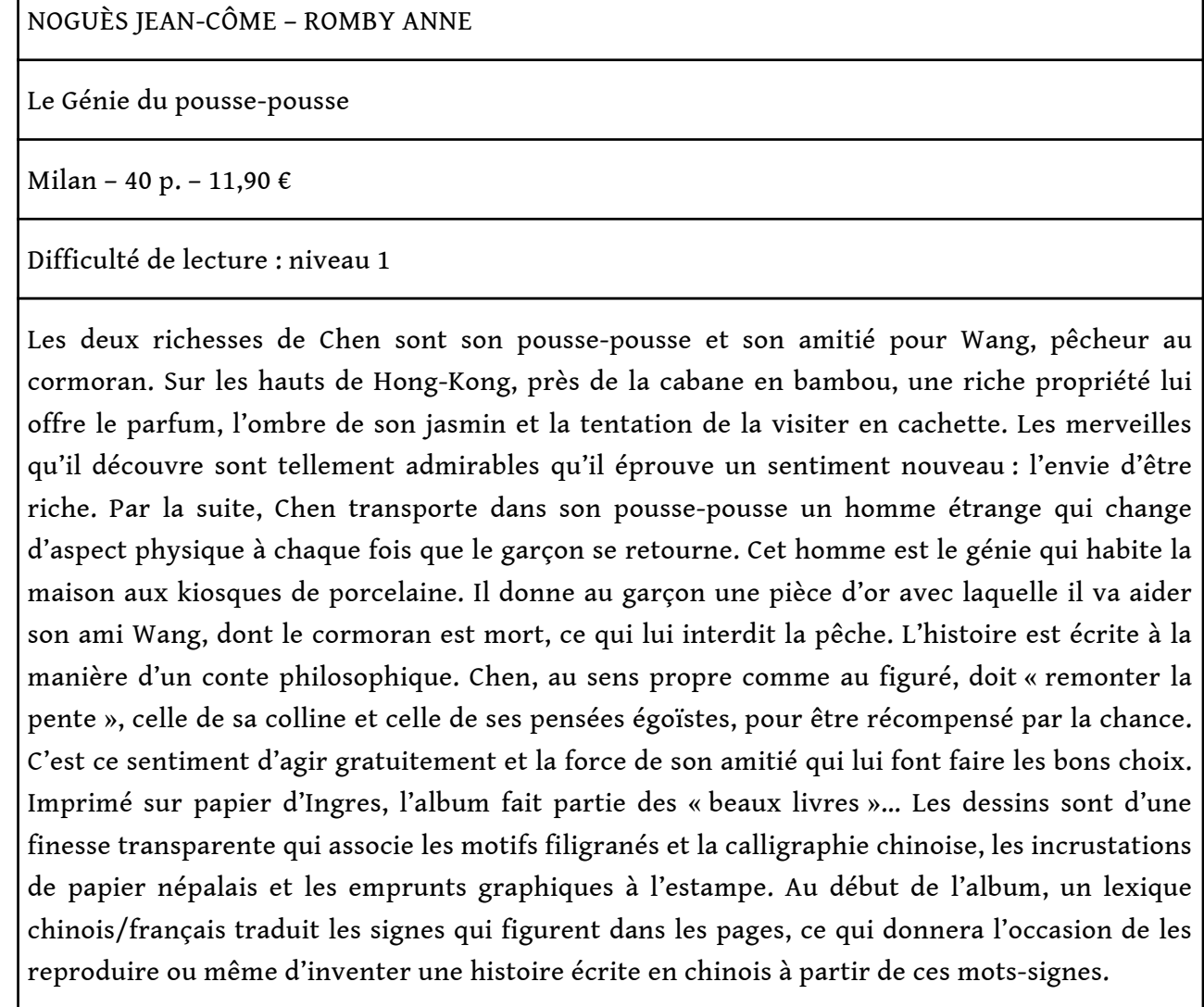

Comme cela a été dit plus haut, l'ouvrage figure sur le site Descola, dans la sélection proposée dans la liste de référence de 2013 pour le cycle 3. Sur ce même site, on trouve toujours la sélection 2007, qui est jugée encore utile pour les enseignants, dans la mesure où « pour chaque œuvre, une notice indiquait les références précises de l'ouvrage, une brève présentation, quelques pistes d'exploitation pédagogique et le niveau de difficulté de lecture ».

\subsection{Présentation « officielle » de l'album}

Voici comment était présenté, dans cette sélection de 2007, l'album qui nous intéresse :

TABLEAU 1. Notice de 2007 présentant Le Génie du pousse-pousse sur le site Éduscol. 
- une injonction de jugement esthétique : il s'agit d'un «beau livre », cela à cause de la qualité du papier et des emprunts à l'art asiatique classique, dont il semble aller de soi qu'on apprécie le raffinement ;

- la mention de l'intérêt esthétique de l'ouvrage, qui permet de diffuser des savoirs, notamment sur l'écriture chinoise.

21 Nous nous intéresserons surtout, dans les lignes qui suivent, aux valeurs portées par l'album, en reconnaissant, bien sûr, que les dimensions esthétique et athrepsique, mentionnées ci-dessus, ne sont pas exemptes, elles-mêmes, de valeurs, même si nous ne développons pas ces points dans ces pages.

\subsection{Analyse du texte}

Pour les besoins de l'article, nous isolons le texte de son péritexte, constitué de la couverture et des images. Le lecteur, lui, certes, perçoit un tout; néanmoins, pour travailler sur la relation texte/image dans l'orientation axiologique de l'album, il nous faut d'abord étudier le texte, qui, s'il n'est pas premier en réception, l'est sans doute en production. Comment le scripteur s'y prend-il pour orienter le texte?

\subsubsection{Remarques génériques}




\subsubsection{Structure narrative et valeurs}

Si l'inscription dans le genre du conte philosophique oriental joue un rôle dans l'orientation de l'album, une manière plus précise de déterminer les valeurs qui circulent dans ce texte est de s'intéresser aux programmes narratifs (PN) du personnage central, Chen. Le programme narratif est l'action par laquelle un sujet transforme un état ${ }^{5}$, le sien ou celui d'un autre sujet (Desmaedt-Everaert, 2003, s'appuyant sur les travaux greimasiens). L'état d'un sujet se définit par sa relation de jonction ou de disjonction avec un objet.

Chen est au départ sans programme narratif. Il vit pauvrement et est heureux. Chen n'a presque rien et son ami Wang est tout aussi pauvre : ce pêcheur n'a qu'un vieux cormoran comme moyen de subsistance. Mais cette pauvreté extrême ne nuit pas au bonheur : la conjonction entre pauvreté et bonheur se trouve fortement marquée au début du récit et est renforcée par la mise en page du texte liée au format album : la première double-page commence en effet par "Chen était pauvre " et la seconde par «Le garçon était heureux ». Mais, à la fin de cette seconde double-page, le bonheur de Chen est quelque peu empreint de tristesse, à cause du malheur de son ami Wang, qui va entrainer la perturbation de la situation initiale "pauvre mais heureux ", où le bonheur est le résultat de la conjonction entre Chen et la quasi-absence de biens matériels.

Deux programmes narratifs se succèdent alors: les deux sont marqués par la quête de la richesse, ou tout au moins d'argent. Mais la comparaison de ces deux programmes narratifs montre une différence. :

- dans le premier cas, l'argent est visé en grande quantité, pour transformer l'état du sujet Chen : « Dans sa tête jusque-là insouciante, il faisait maintenant des calculs. Il placerait cet argent à un taux élevé. Il en prêterait une partie à Wang, mais contre un bon intérêt. Et il aurait, lui aussi, des kiosques de porcelaine »;

- dans le second cas, l'argent est visé, mais en moindre quantité, et surtout avec un autre objectif, altruiste, celui de transformer l'état de Wang, en lui achetant un cormoran pour remplacer celui qu'il vient de perdre et qui était son gagne-pain. Chen est lié à Wang par un engagement d'amitié : «Si j'ai de l'argent ce soir, je te le donnerai. » Réussir son programme narratif revient à satisfaire à cet engagement.

Le premier PN ayant échoué, Chen renonce à sa première quête, celle de la richesse, qui se faisait au détriment de l'amitié : « Le temps des mauvais calculs était bien fini. Noyés dans le port, les désirs d'égoïste en puissance, les rêves de richesse gagnés au prix de l'amitié. »

Mais le second PN réussit, réussite symbolisée par la pièce d'or donnée à Chen par le génie (passages 18 et 19 en annexe).

La structure de ce conte, en fait, fait se succéder deux apprentissages, l'un négatif, l'autre positif : «En juxtaposant au moins deux histoires d'apprentissage dont chacune réalise une des deux variantes, le récit souligne l'opposition entre elles et marque, par le fait même de leur juxtaposition, la valeur positive ou négative de chacune » (Suleiman, 1983 : 105). Il ressort de la lecture du conte que ce qui est valeur pour Chen, c'est l'amitié plus que l'argent en lui-même, ainsi que la pauvreté dans laquelle il restera par ailleurs.

Les deux PN se différencient également par le type d'action qu'ils entrainent. Comme la fée dans Les Fées de C. Perrault (1697), le personnage merveilleux prend deux aspects: celui d'un client jeune, beau, léger et facile à transporter dans le premier PN; et celui d'un client très lourd, voulant gagner le sommet d'une montagne dans le second PN. Pour 
gagner l'argent destiné à Wang, Chen souffre : « La sueur lui brûlait les yeux. Les muscles de son cou étaient près de craquer tant le malheureux serrait les mâchoires pour se concentrer sur l'effort. »

L'amitié (Chen est le premier mot du texte et Wang en est le dernier mot), la pauvreté, le désintéressement, sont des valeurs pour Chen, mais aussi l'effort, le travail qui sont préférables à la facilité. La peine, l'effort, la souffrance physique sont récompensés, alors que céder à la facilité (prendre en charge un client léger) n'a mené nulle part: la rédemption est ainsi valorisée.

\subsubsection{Identification au personnage central et valeurs}

Outre l'analyse de la structure du conte, l'identification au personnage de Chen est nécessaire. En effet, comme le remarque S. R. Suleiman (1983 : 91-92) à propos du roman à thèse, le scripteur, pour faire partager au lecteur des valeurs, a à sa disposition plusieurs procédés, dont celui-ci :

"C'est l'histoire elle-même, en tant qu'elle est vécue comme expérience, (comme transformation) par un sujet à travers le temps. Nous retrouvons ici la structure d'apprentissage, qui est précisément une structure au niveau de l'histoire : le sujet fictif qui "vit" l'histoire a sa contrepartie dans le sujet réel qui la lit. L'effet persuasif d'une histoire d'apprentissage à thèse passe par l'identification virtuelle du lecteur avec le protagoniste. Dans la mesure où celui-ci évolue vers une position euphorique, le lecteur est incité à le suivre dans la bonne voie. Le bonheur du protagoniste fonctionne comme preuve et garant des valeurs qu'il affirme. Si le protagoniste finit mal, son échec sert également de leçon ou de preuve, mais cette fois-ci a contrario : le destin du protagoniste permet au lecteur de voir la mauvaise voie, sans la suivre."

L'itinéraire de Chen ne fonctionnera pas comme garant de ses valeurs si le lecteur ne s'identifie pas à lui. Il est question ici de la part du lecteur que V. Jouve (1992) appelle le lisant, qui se laisse prendre à l'illusion référentielle et toucher affectivement par les péripéties vécues par les personnages.

Pour V. Jouve, la projection du lecteur dans une figure romanesque est l'effet des trois codes constituant le système de sympathie. Le premier de ces codes joue sur le choix du point de vue narratif: le lecteur s'identifie à qui occupe dans le texte la même place que lui, à celui du point de vue duquel les événements sont découverts. Le code affectif, lui, règle l'accès à l'intimité de l'acteur romanesque par des techniques narratives, notamment le mode de représentation de sa vie psychique ${ }^{6}$, et par la présence de thèmes comme l'enfance, les rêves, la souffrance, qui assurent une plus grande proximité entre lecteur et personnage, une projection affective. Quant au code culturel, contrairement aux deux précédents qui dépendent étroitement de l'organisation textuelle, il repose uniquement sur le sujet lecteur qui, en fonction de son axiologie propre, valorise ou dévalorise le personnage, se projette idéologiquement en lui ou non.

Pour le code narratif, les événements sont découverts du point de vue de Chen. Par exemple, la description du jardin de la maison voisine de celle de Chen est faite du point de vue, représenté (Rabatel, 2004, 2005), de ce dernier :

«Chen escalada le mur et resta muet d'admiration devant ce qu'il découvrit.

A ses pieds s'étendait un jardin merveilleux. Toutes les fleurs de l'Asie y mêlaient leurs couleurs. Des kiosques de porcelaine se reflétaient dans des pièces d'eau claire, bordées de sentiers recouverts d'un gravier blanc. Le garçon sauta à bas du 
mur et se risqua le long des pelouses, où tombaient, un à un, des pétales de magnolia.

Dans les kiosques, des mots de bienvenue étaient peints en rouge vif sur du papier de soie.

Des mots qui offraient les gâteaux aux amandes, et les confitures de roses, et les liqueurs, et les fruits servis dans des corbeilles d'argent, sur des tables de laque.

Chen goûta de ces douceurs délicieuses qui lui firent tourner la tête puis il s'enfuit en courant. » personne, la voix du narrateur anonyme joue un rôle important dans l'axiologisation générale, tant il est vrai que, comme l'écrit S. R. Suleiman (1983 : 90),

« dans la mesure où le narrateur se pose comme source de l'histoire qu'il raconte, il fait figure non seulement d'“auteur" mais aussi d'autorité. Puisque c'est sa voix qui nous informe des actions des personnages et des circonstances où celles-ci ont lieu, et puisque nous devons considérer - en vertu du pacte formel qui, dans le roman réaliste, lie le destinateur de l'histoire au destinataire - que ce que cette voix raconte est "vrai", il en résulte un effet de glissement qui fait que nous acceptons comme "vrai" non seulement ce que le narrateur nous dit des actions et des circonstances de l'univers diégétique, mais aussi ce qu'il énonce comme jugement ou comme interprétation. Le narrateur devient ainsi non seulement source de l'histoire mais aussi interprète ultime du sens de celle-ci. »

Cette voix est omnisciente. Cela est particulièrement perceptible dans le fait qu'elle s'exhibe en tant qu'instance narratrice ( 'C'est ainsi que commencent les aventures, par une idée inattendue, un petit rien qui vous entraîne au loin ») et qu'elle livre accès aux pensées et aux sentiments de Chen : «Le garçon était heureux »; « Pour la première fois, il se trouva malheureux $"$.

D'un point de vue axiologique, cette voix narratoriale se manifeste ici de façon assez nette par des marques évaluatives disséminées ou condensées dans des seconds plans plus ou moins longs au présent ou à l'imparfait. Voici quelques exemples de ces marques évaluatives :

(1) «Le garçon était heureux.»

(2) «Le temps des calculs était bien fini. Noyés dans le port, les désirs d'égoïste en puissance, les rêves de richesse gagnés au prix de l'amitié. » l'état de pauvreté de Chen (1), en l'associant au bonheur, qui marque nettement sa préférence pour l'amitié sur l'amour de la richesse pour soi (2).

On notera trois caractéristiques de ces marques évaluatives narratoriales :

- leur redondance contribue au guidage axiologique du lecteur ; 
- elles renforcent ce qui est mis en place par la structure narrative ;

- elles servent une distribution binaire des valeurs : la pauvreté est valorisée par rapport à la richesse, l'altruisme est valorisé par rapport à l'égoïsme, l'amitié est valorisée par rapport à l'intérêt, le travail est valorisé par rapport à la facilité. personnages porte-paroles du narrateur. On peut noter ici que les valeurs qui se manifestent dans les propos et les pensées rapportés de Chen, qu'elles soient représentées, racontées ou assertées (Rabatel, 2004, 2005) sont orientées de la même façon que celles du narrateur. Le lecteur est donc guidé de façon efficace pour remonter jusqu'aux valeurs du scripteur, qui sont celles du texte de l'album.

\section{Analyse du rapport texte/image dans deux doubles- pages}

50 Il existe un certain nombre d'ouvrages et d'articles qui proposent des analyses de la relation texte-image, sur laquelle travaillent de plus en plus de chercheurs, du fait du développement contemporain des médias multimodaux, où image, texte et son peuvent être présents simultanément. Cinéma, bande dessinée, publicité se retrouvent à l'ordre du jour (dans les années 70, la didactique s'y intéressait déjà) et sont rejoints, dans leur multimodalité, par les sites web. Si R. Barthes (1964) a été sans doute le précurseur le plus connu des travaux abordant la question des relations texte/image, de nombreuses autres références peuvent être convoquées aujourd'hui. On peut citer, entre autres, les travaux typologiques, non traduits en français, d'E.E. Marsh et M.D. White (2003) et de R. Martinec et A. Salway (2005), ainsi que ceux de J.-M. Klinkenberg (2008), les textes réunis par J.-L. Tilleuil (2005) ${ }^{7}$, l'ouvrage d'A. Beguin-Verbrugge (2006). Si la relation texte/image, dans la publicité, les œuvres littéraires pour adultes, les manuels scolaires, donne lieu à un certain nombre d'études, il n'en est pas de même pour l'album, tout au moins en France. Certes, certains albums ont été étudiés dans cette perspective, mais, à notre connaissance, seuls les travaux de S. Van der Linden (2003, 2006, 2007, 2008) proposent une perspective synthétique dans ce domaine ${ }^{8}$.

51 Nous prendrons comme unité d'analyse la double-page. Comme le souligne A. BéguinVerbrugge (2006: 177), « l'habitude de structurer l'information sur une double-page [...] vient d'une prise de conscience des spécificités de la lecture enfantine (faible empan visuel, lenteur du décodage, limites de la mémoire immédiate et de l'attention) et du rôle de l'image comme aide à la construction du sens ». Comme, dans le cadre de cet article, nous ne pouvons analyser complètement l'album dans le rapport texte/image, nous avons 
choisi trois doubles-pages qui nous permettront de dégager quelques caractéristiques du rôle du rapport texte/image dans l'orientation générale de l'album :

- la double-page initiale (annexe, extrait 1), parce que, correspondant à l'état initial du conte, elle installe l'univers de celui-ci ;

- la double-page (annexe, extrait 5), où se joue le premier basculement de Chen, qui, rêvant de richesse, perd sa joie de vivre ;

- la double-page (annexe, extrait 16) où se manifeste le second revirement de Chen : après s'être rendu compte de la vanité de sa quête de la richesse, il veut gagner de l'argent pour Wang, en exerçant sa profession.

Après avoir défini le type de relation texte/image à l'œuvre dans cet album de façon générale, nous illustrerons de façon plus précise une caractéristique de ce rapport par l'analyse successive de ces trois doubles-pages.

\subsection{Une image et un texte complémentaires}

Traditionnellement (Van der Linden, 2008), le rapport texte/image est décrit selon trois pôles: redondance, complémentarité, dissociation (ou opposition). Mais pour J.-M. Klinkenberg (2008), que nous suivrons sur ce point, la redondance parfaite n'existe pas: elle ne peut être que partielle, les sémiotiques linguistique et iconique n'ayant pas les mêmes potentialités. Par exemple, la sémiotique iconique permet la présentation simultanée des objets, ce que ne permet pas la sémiotique linguistique; en revanche, celle-ci autorise l'expression de modalités ou de quantifications inaccessible à la sémiotique iconique. On préfèrera donc parler de complémentarité plutôt que de redondance.

Les places respectives qu'occupent, dans l'espace de l'album, le texte et l'image, sont les premières à jouer un rôle dans cette complémentarité. Comme le note $\mathrm{S}$. Van der Linden (2008: 51), "l'album ne montre pas des types d'organisation faciles à circonscrire. Pourtant, la mise en page conditionne en grande partie le discours véhiculé. En fonction de la narration ou de l'effet recherché, l'illustrateur positionne les images ou les textes de manière à tirer parti de son support ». La place de l'image est signifiante. Nous prendrons l'exemple de la première double-page de l'album. L'œil du lecteur, du fait de l'apparition, dans l'espace de cette double-page, à gauche d'un texte avec une image qui s'y insère, et à droite d'un dessin principal, effectue des va-et-vient de l'un à l'autre, même si, le texte étant sur la page de gauche, il est en quelque sorte mis en avant. Le lecteur fait ainsi sens dans l'interaction entre texte et image.

L'image qui occupe toute la page de droite concentre un certain nombre d'informations délivrées par le texte et concernant la vie du personnage principal: une représentation de Chen, le bol de riz, le chapeau, la cabane, le pousse-pousse, la baie de Hong-Kong... Mais ce n'est pas pour autant que l'image est redondante par rapport au texte. Si elle semble concentrer tous les éléments évoqués successivement par le texte, le texte seul précise le nom du personnage, le lieu où cela se passe, la relation entre le personnage et le lieu, un détail vestimentaire (le chapeau) concernant le personnage. L'image, elle, apporte son complément d'informations : l'époque, celle de la Chine traditionnelle, l'âge de Chen, le caractère souriant de celui-ci... Les signes iconiques de l'image principale se référant au texte sont nombreux. Mais chacun des deux sous-énoncés, le texte et l'image, offre donc nécessairement un surcroit d'information par rapport à l'autre. 


\subsection{L'effet axiologique proleptique de la première image principale}

Chacune des doubles-pages analysées dans les paragraphes suivants est représentée par un schéma indiquant les places respectives du texte, de l'image principale (nous entendons par là l'image dont la taille est la plus importante, et nous l'appellerons désormais IP) et des images secondaires (désormais IS).

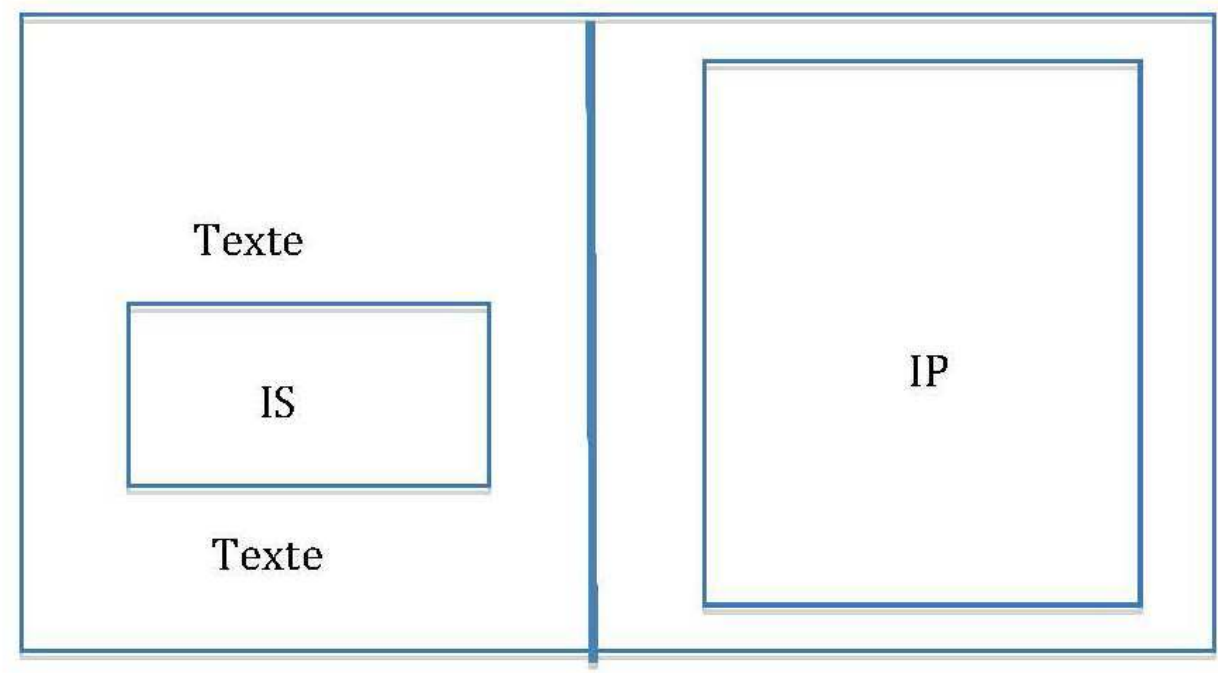

FIgURE 1. Double-page, extrait 1 (annexe).

Dans cette double-page, l'IP interpelle particulièrement. Tout y semble à sa place : un plan de demi-ensemble situe le personnage, de face, dans son environnement proche, qui reprend les éléments que l'on trouve dans les dessins ou estampes orientaux traditionnels, la nature, les objets de la vie quotidienne. Cette image donne une vision positive de l'état de pauvreté. En effet, le paysage est idyllique, il fait beau, la mer se voit au loin avec ses jonques, la nature souriante est exubérante, quoique contenue par le mur d'une propriété. Les couleurs sont chaudes, ocre, brun et brique pour les éléments minéraux, vert pour les arbres et le toit de la cabane de Chen. Ce dernier est en tenue traditionnelle blanche, simple et immaculée, blanc que l'on retrouve dans le toit du pousse-pousse et dans les fleurs de jasmin. La scène entière est douce et lumineuse, sans doute mise en valeur par l'éclairage diffus qui la caractérise. L'abondance de lignes courbes, associées à la douceur, celles du chapeau, du mont sur la mer au loin, des bols en bois, du dessin du pousse-pousse, constitue un autre signe plastique participant à la connotation positive de l'environnement du personnage. Le personnage de Chen est souriant. Tout un jeu de correspondances entre couleurs, textures, lumière, concourt à un phénomène de synesthésie (Joly, 2011) qui permet aux signes iconiques et plastiques ${ }^{9}$ d'entrer en conjonction pour associer pauvreté et bonheur. En effet, le dénuement évoqué dans le texte ("Chen possédait peu de choses», " une cabane, c'est beaucoup dire ») est celle d'un robinson sur une ile paradisiaque, et les choix esthétiques de l'illustratrice, l'association faite du plastique et de l'iconique se référant à la peinture traditionnelle chinoise, idéalisent la pauvreté. 

rapport à la page de texte suivante, qui commence par «Chen était heureux». Elle constitue aussi une prolepse du dénouement, puisque le récit se termine par un retour à la situation initiale, après un passage par l'envie d'en sortir pour atteindre la richesse. D'ailleurs cette première image est une image de stabilité. Le lecteur adulte de l'album y retrouvera le thème des dessins chinois, les fleurs, la façon dont la nature est représentée, le souci de peindre la vie quotidienne.

\subsection{L'opposition comme moyen d'axiologiser l'album}

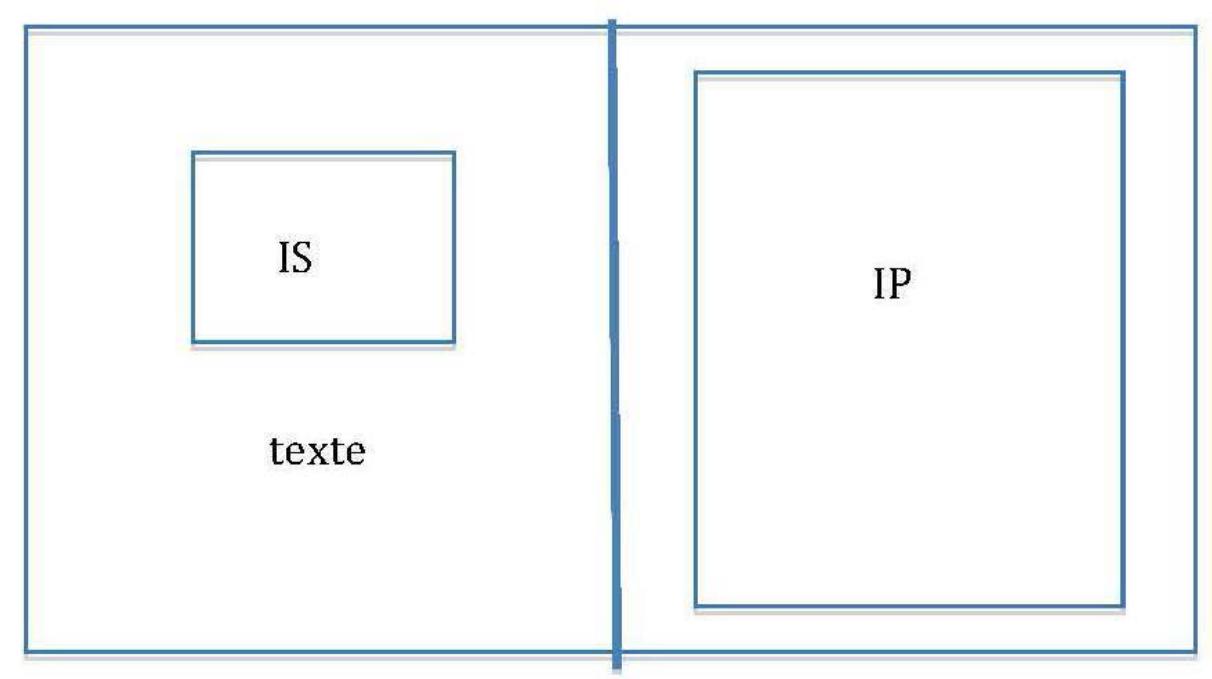

FIgURE 2. Double page, extrait 5 (annexe).

L'analyse de la double-page de l'album correspondant à l'extrait 5 de l'annexe permet d'illustrer une autre manière d'axiologiser le texte, les jeux d'opposition. L'IS et l'IP reprennent l'opposition textuelle entre la modestie heureuse de Chen auparavant et le désir de luxe né de sa visite de la demeure du génie. L'opposition se joue à deux niveaux.

Tout d'abord, l'IP entre en opposition avec l'IP commentée précédemment de plusieurs manières. Le cadre naturel figuratif a disparu pour laisser place à un cadre stylisé, non figuratif, où l'on retrouve cependant les couleurs chaudes de la première image. Là où il y avait ouverture, sur les arbres, sur la mer, il y a maintenant fermeture dans une forme symétrique. Le personnage de Chen est traité tout à fait différemment. Il apparait de face encore une fois, mais sans le chapeau qu'il porte habituellement dans l'exercice de son métier. Il est vêtu de ce qui ressemble à une robe impériale d'apparat, de celles qui apparaissent dans certains manuscrits chinois ${ }^{10}$. Son visage n'est plus souriant, mais impassible, quoiqu'une larme perle à son œil gauche. Il est sans pieds ni mains, les bras tournés vers le bas, en une position figée, et ressemble plus à une figure divine qu'à un simple tireur de pousse-pousse. Au-dessus de sa tête, une coupe de porcelaine richement décorée est représentée légèrement du dessous, pour que le lecteur puisse voir les motifs qui l'ornent. Comme s'échappant de cette coupe, des caractères chinois se répètent: 
multitude est un mot qui revient ainsi quatre fois. D'autres caractères chinois, signifiant respectivement esprit, contradiction, cascade, parfum, étoffe, soie, désir, pouvoir, souffle, vide, interroger ${ }^{11}$, auréolent la tête de Chen, connotant l'orientalisme du conte mais aussi indiquant la confusion et le trouble du personnage. Pour résumer, cette image est un peu moins figurative que celle que nous avons commentée dans le paragraphe précédent, et plus le lecteur se trouve au cœur de l'aventure de Chen, plus la dimension plastique l'emportera sur la dimension iconique.

Une autre opposition se manifeste sur la même double-page : l'IS, placée avant le texte, si bien que le regard du lecteur s'y pose d'abord, ne serait-ce qu'un instant, montre l'humble bol de bois dont Chen se contentait auparavant, et ce bol, relégué dans une IS, représenté cette fois en contre-plongée, entre doublement en opposition avec la coupe de porcelaine représentée sur l'IP.

Pour conclure sur ce point, IP et IS interagissent et reprennent ainsi parfaitement l'opposition du texte, entre simplicité et bonheur, et richesse et malheur, et, ainsi, la reprenant, la renforcent, par le moyen de la mise en scène du personnage et des objets, et contribuent à l'orientation générale des valeurs de l'album.

\subsection{L'image comme visualisation des pensées de personnage}

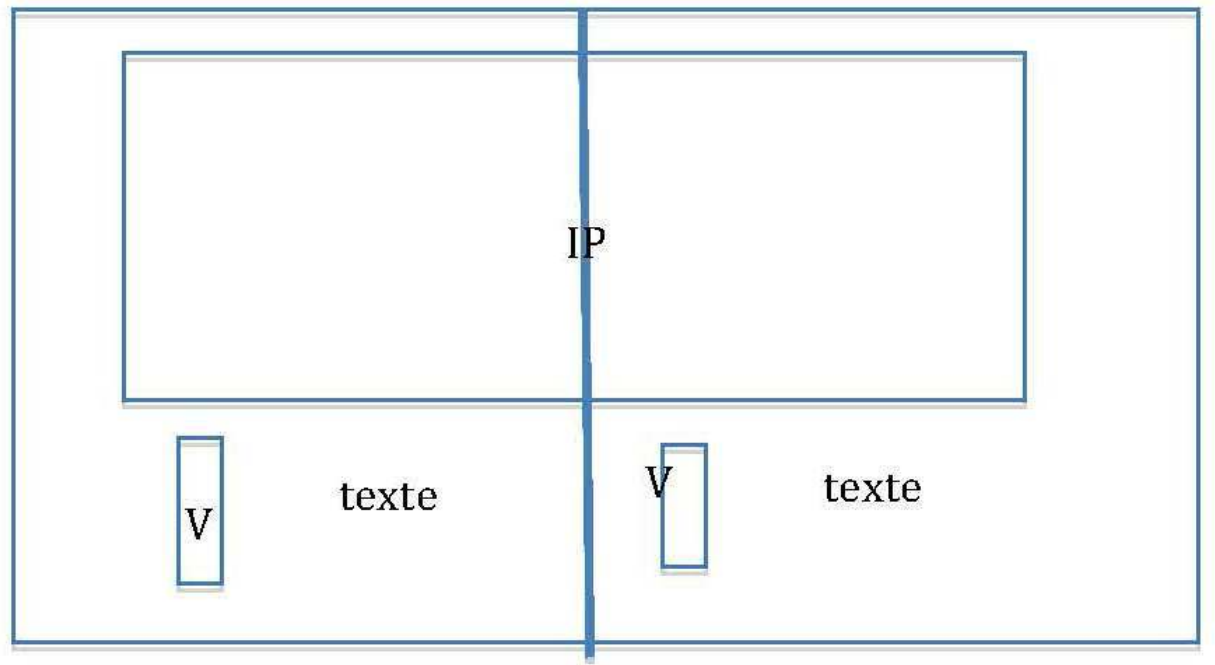

FIgURE 3. Double-page, extrait 16 (annexe).

Dans cette double-page correspondant à l'extrait 16 (annexe), on voit une IP placée avant le texte et occupant toute la double-page, et deux petites images, s'apparentant à des vignettes, au début de chaque zone de texte.

L'IP est à mettre en relation avec une IP précédente, avec laquelle elle fonctionne en opposition, selon le procédé signalé ci-dessus. Cette IP précédente, représentant la même scène de rue, était située après le texte. Chen y prenait en charge un jeune et riche client (une apparence prise par le génie) dans l'indifférence générale des passants. Un enfant jouait dans la rue, derrière le pousse-pousse, et Chen courait légèrement, tout à la joie de gagner facilement beaucoup d'argent. L'IP correspondant à l'extrait 16, elle, est placée avant le texte, si bien qu'elle est offerte aux yeux du lecteur avant celui-ci. Le client est 
beaucoup plus lourd, si bien qu'il déforme le pousse-pousse et que Chen est courbé vers le sol par l'effort. Tous les passants sont hilares en voyant le tableau de ce jeune garçon tractant un imposant personnage, et l'autre jeune garçon, qui, dans l'image antérieure correspondante, jouait dans la rue, ici aide comme il peut le pousse-pousse à avancer. Dans le texte, tout traduit l'effort, la description du pousse-pousse ("le rotin gémit ", «l'essieu fléchit», les roues sont comme "clouées au sol») comme l'évocation de Chen (" tendit ses jarrets maigres ", " par un effort violent arracha le pousse-pousse », « la sueur lui brûlait les yeux. Les muscles de son cou étaient près de craquer »). L'IP fait de même, renforçant ainsi le thème de la rédemption.

Si on retrouve dans cette IP le jeu d'opposition mentionné précédemment, ce qui nous intéresse surtout, dans cette double-page, ce sont les deux vignettes qui figurent en vis-àvis du texte. Ces dernières, en effet, expliquent, développent, en les illustrant, les pensées rapportées de Chen : «Wang sera content ». Elles rendent présents à l'esprit du lecteur le visage de Wang, souriant, et le cormoran que Chen a fait le serment de remplacer. Les deux vignettes sont ornées de graines volantes de pissenlit, symboles de légèreté. Elles concrétisent les valeurs qui sont celles de Chen lors de ce deuxième PN, l'amitié et l'altruisme.

Les romans, les films, les albums peuvent susciter des réactions et des questions qui touchent aux valeurs. Mais comme le souligne A. Petitjean (2014), leur fonction n'est pas de servir de modèles ou de contre-modèles. Ce qui nous a intéressé ici réside plus dans la façon dont les valeurs sont mises en place dans les albums, supports souvent proposés aux élèves à l'école primaire, que dans ces valeurs elles-mêmes, qui peuvent être très variables d'un ouvrage à l'autre. L'important, pour nous, est d'armer les enseignants et les élèves pour qu'ils puissent les repérer. Si A. Rabatel a proposé, en 2005, un ouvrage didactique sur cette question plutôt destiné aux enseignants du secondaire, la même réflexion reste à poursuivre pour l'école primaire.

L'album fait partie des supports présents à l'école, de la maternelle au cycle 3. La didactisation des valeurs, dans la lecture d'albums, passe par l'étude, du moins par les enseignants, de la façon dont texte et image interagissent pour mettre en place ces valeurs. C'est cette étude que nous avons initiée dans ces lignes. Nous avons ainsi mis en avant trois fonctions de la relation texte/image dans l'orientation axiologique du Génie du pousse-pousse : la dimension proleptique de l'image sur le texte, le renforcement des jeux d'oppositions textuels par ceux des images et enfin le rôle de l'image dans la visualisation de ce qui peut être plus ou moins inféré, à partir du texte, des pensées de personnages.

Cette analyse est très partielle et, de plus, porte sur un seul album. Mais il ne s'agit que du début de recherches qui exploreront de façon plus systématique les relations texte/image dans l'orientation des albums, et qui s'intéresseront aussi à la façon dont, dans les classes de l'école primaire, on peut mettre en place des modalités d'approche des albums en prenant en compte cette dimension. 


\section{BIBLIOGRAPHIE}

BARTHES, R. (1964) : « Rhétorique de l'image », Communications, 1, vol. 4, p. 40-51.

BAUTIER, E. \& Rayou, P. (2009) : Les inégalités d'apprentissage. Programmes, pratiques et malentendus scolaires, Paris, Presses universitaires de France.

BÉGUIN-VERBRUGGE, A. (2006) : Images en texte, images du texte. Dispositifs graphiques et communication écrite, Villeneuve-d'Ascq, Presses universitaires du Septentrion.

Chirouter, E. (2007) : Lire, réfléchir et débattre à l'école. La littérature de jeunesse pour aborder des questions philosophiques, Paris, Hachette.

- (2012) : «Philosopher avec les enfants dès l'école élémentaire grâce à la littérature : analyse d'un corpus de trois années d'ateliers de philosophie en cycle 3 ", Recherches en éducation, 13, p. 32-42.

- (2014) : «Si j’étais invisible », Cahiers pédagogiques, 513, p. 38-39.

DiAS-CHIARUTTINI, A. (2007) : « Du débat interprétatif à l'école ? », Recherches, 46, p. 151-166.

EVERAERT-DESMEDT, N. (2003) : « Scène de rue au Brésil. Analyse et exploitation d'un album pour enfants », in : P. Marillaud \& R. Gauthier (éds), Les langages de la ville, actes du $23^{\mathrm{e}}$ colloque d'Albi Langages et Significations, Toulouse, Université de Toulouse-Le Mirail, p. 1-15.

GALLAND, A. (1965 [1704]) : Les mille et une nuits, vol. 1, Paris, Garnier-Flammarion.

GENETTE, G. (1999) : Figures IV, Paris, Éd. Le Seuil.

GREIMAS, A.-J. (1983 [1973]) : «Un problème de sémiotique narrative : les objets de valeur », repris in : A. J. Greimas, Du sens II. Essais sémiotiques, Paris, Éd. Le Seuil, p. 19-48.

GREIMAS, A. J. \& COURTES, J. (1993) : Sémiotique. Dictionnaire raisonné de la théorie du langage, Paris, Hachette.

HAMON, P. (1984) : Texte et idéologie, Paris, PUF.

JOLY, M. (2011[1993]) : L'image et les signes, Paris, A. Colin.

JoUVE, V. (1992) : L'effet-personnage dans le roman, Paris, PUF.

KLINKENBERG, J.-M. (2008) : « La relation texte-image. Essai de grammaire générale », Bulletin de la Classe des Lettres, $6^{\mathrm{e}}$ série, t. XIX, p. 21-79 (en ligne : http://gemca.fltr.ucl.ac.be/docs/ cahiers/20090128_Klinkenberg.pdf, consulté le 05/04/13).

- (2011) : «Conclusions. De la valeur d'échange à la valeur éthique, en passant par la valeur de survie », Semen, 32, p. 161-174.

Leclaire-halté, A. \& masseron, C. (coords) (2003) : « Textes et valeurs », Pratiques, 117-118.

LeClaire-halté, A., AUdoin N., PATÉ, S., RONDELLI, F. \& SPECognA, A. (2009) : « Le rapport texte/image dans le traitement de l'album au cycle 3 : quelques remarques sur les pratiques enseignantes », Diptyque, 17, p. 115-130.

LECLAIRE-halté, A. \& RondelLi, F. (2015) : « Un album satire de la téléréalité au cycle 3 : un choix problématique ", Spirale, 55, p. 21-30. 
LELEUX, C. (2009) : « La discussion à visée philosophique pour développer le jugement moral et citoyen? ", Revue française de pédagogie, 166, p. 71-87.

MARSH, E. E. \& White, M. D (2003) : «A Taxonomy of Relationships between Images and Text : Additional Examples Illustrating its Application », Journal of Documentation, p. 647-672.

MARTINEC, R. \& SAlWAY, A. (2005) : « A System for Image-Text Relations in New (and Old) Media », Visual Communication, 3, vol. 4, p. 337-371.

NicolajeVA, M. (2012): « Reading Other People's Minds Through Word and Image », Children's Literature in Education, 43, p. 273-291.

NicolajeVA, M. \& scott, C. (2000) : « The Dynamics of Picturebook Communication », Children's Literature in Education, 4, vol. 31, p. 225-239.

- (2001) : How picture books work, New York, Garland.

NIÈRES-CHEVREL, I. (2011) : « La littérature d'enfance et de jeunesse entre la voix, l'image et l'écrit (en ligne : http://www.vox-poetica.org/sflgc/biblio/nieres-chevrel.html, consulté le 02/05/14).

NOGUÈs, J.-C. (illus. par Romby A.) (2011 [2001]) : Le Génie du pousse-pousse, Paris, Milan.

PERRAULT, C. (1697) : Les Fées, Paris, C. Barbin.

PERRIN, J.-F. (2005) : «L'invention d'un genre littéraire au XVIII ${ }^{\mathrm{e}}$ siècle », Féeries, 2 , p. 9-27.

PEYTARD, J. (1982) : «Instances et entailles du texte littéraire », in : Littérature et classe de langue, Paris, Hatier/Credif, p. 139-150.

PETITJEAN, A. (1991) : « Contribution sémiotique à la notion de "genre textuel" ", in : G. Gréciano \& G. Kleiber (éds), Systèmes interactifs. Mélanges en l'honneur de Jean David, Metz, Centre d'analyse syntaxique-Université de Metz (coll. « Recherches linguistiques »), p. 349-373.

- (2014) : « 40 ans d'histoire de la "lecture littéraire" au secondaire à partir de la revue Pratiques », Pratiques, 161-162 (en ligne : http://pratiques.revues.org/2155, consulté le 10/01/15).

RABATEL, A. (2004) : Argumenter en racontant, Bruxelles, De Boeck.

- (2005) : « La construction inférentielle des valeurs », Cahiers de narratologie, 12, p. 1-17.

SUlEIMAN, S. R. (1983) : Le roman à thèse, Paris, Presses universitaires de France.

SERAFINI, F. (2010) : « Reading Multimodal Texts : Perceptual, Structural and Ideological Perspectives ", Children's literature in Education, 41, p. 85-104.

SIPE, L. R. (2012) : « Revisiting the Relationships between Text and Pictures », Children's Literature in Education, 43, p. 4-21.

TAUVERON, C. (2002) : Lire la littérature à l'école, Paris, Hatier.

- (2004) : « La lecture comme jeu, à l'école aussi », in: C. Tauveron (éd.), La lecture et la culture littéraire au cycle des approfondissements, Versailles, Centre régional de documentation pédagogique de Versailles, p. 32-40.

TILLEUIL, J.-L. (2005) : Théories et lectures de la relation image-texte, Cortil-Wodon, Éd. modulaires européennes.

VAN DER LINDEN, S. (2003) : "L'album entre texte, image et support », La Revue des livres pour enfa nts, 214, p. 59-68.

- (2006) : Lire l'album, Paris, L'Atelier du poisson soluble.

- (2007) : «L'articulation des messages visuels et linguistiques dans l'album de jeunesse contemporain ", in : J.-L. Tilleuil \& M. Watthee-Delmotte (dirs), Texte, Image, Imaginaire, actes du premier colloque organisé dans le cadre des échanges U.C.L.-UMASS (1999-2006), Paris, 
Éd. L'Harmattan, p. 353-359.

- (2008) : « L'album, le texte et l'image », Le Français aujourd'hui, 161, p. 51-58.

\section{ANNEXES}

\section{Annexe. Texte du Génie du pousse-pousse}

(1) Chen possédait peu de choses.

D'abord un pousse-pousse en rotin qui était sa vraie richesse, car il lui permettait de gagner de quoi s'acheter un bol de riz et, quelquefois, un petit pâté de viande au gingembre.

Puis un grand chapeau qui lui servait aussi de parapluie.

Et puis, en haut d'une colline, dominant la baie de Hong Kong, une cabane de bambous.

Une cabane, c'est beaucoup dire. La maison de Chen était faite de trois planches et d'une brassée de feuillage.

Mais elle était adossée au mur d'enceinte d'une belle propriété. De ce mur retombaient des branches de jasmin qui, au temps des fleurs, donnaient à Chen une ombre parfumée./

(2) Le garçon était heureux. Toute la journée, il conduisait son pousse-pousse dans les rues de la ville. Le soir venu, il allait bavarder avec son ami Wang, le pêcheur au cormoran.

Wang se plaignait invariablement que son oiseau vieillissait.

- Il faut que je pêche beaucoup de poissons pour pouvoir m'acheter un autre cormoran. Mais, si je n'ai plus de cormoran, comment arriverai-je à pêcher des poissons?

Problème insoluble. Chen ne trouvait pas de réponse. Wang soupirait.

Un soir, en retournant à sa cabane, Chen eut envie de cueillir un rameau de jasmin pour chasser l'impression de tristesse laissée par son ami.

C'est souvent ainsi que commencent les aventures, par une idée inattendue, un petit rien qui vous entraîne au loin./

(3) Chen escalada le mur et resta muet d'admiration devant ce qu'il découvrit.

A ses pieds s'étendait un jardin merveilleux. Toutes les fleurs de l'Asie y mêlaient leurs couleurs. Des kiosques de porcelaine se reflétaient dans des pièces d'eau claire, bordées de sentiers recouverts d'un gravier blanc.

Le garçon sauta à bas du mur et se risqua le long des pelouses, où tombaient, un à un, des pétales de magnolia./

(4) Dans les kiosques, des mots de bienvenue étaient peints en rouge vif sur du papier de soie.

Des mots qui offraient les gâteaux aux amandes, et les confitures de roses, et les liqueurs, et les fruits servis dans des corbeilles d'argent, sur des tables de laque. 
Chen goûta de ces douceurs délicieuses qui lui firent tourner la tête puis il s'enfuit en courant./

(5) Comme elle tournait, la pauvre tête de Chen, quand, de nouveau, il franchit le mur ! Jusqu'à ce jour, il s'était contenté d'un bol de bois, satisfait qu'il était quand il pouvait le remplir de riz.

Et voilà qu'il désirait maintenant une coupe de jade.

Le petit pâté des jours de fête, naguère dévoré en riant, lui serait désormais pauvre chère après des nourritures dont, la veille encore, il ne soupçonnait même pas l'existence.

Pour la première fois, il se trouva malheureux./

(6) Il tira son pousse-pousse et se dirigea vers la ville basse. Il allait pensivement quand, passant près de l'entrée de la belle propriété, il s'entendit héler.

Un jeune homme lui faisait signe d'approcher. Il était magnifiquement vêtu d'une robe couleur de soleil sur laquelle s'embrasaient des pivoines. Surtout, il était très beau, avec un teint clair, une natte brillante et des mains fines qu'il glissa précautionneusement dans ses manches.

Mais qu'il avait l'air frêle ! De loin, on aurait pu le prendre pour un enfant./

(7) «Il est riche et sans doute léger, pensa Chen. Double bonne affaire!»

- Conduis-moi au port, dit le jeune homme d'une voix douce.

$\mathrm{Au}$ port ! Le long des rues qui descendaient à la mer ! Il n'y aurait qu'à se laisser porter par les brancards et à poser le pied de loin en loin pour rebondir toujours plus haut./

(8) « Triple bonne affaire ! pensa Chen. Et je triplerai le prix », se promit-il aussitôt.

Dans sa tête jusque-là insouciante, il faisait maintenant des calculs. Il placerait cet argent à un taux élevé. Il en prêterait une partie à Wang, mais contre un bon intérêt.

Et il aurait, lui aussi, des kiosques de porcelaine.../

(9) Il était si absorbé dans ses pensées qu'il ne se rendit pas compte de la vitesse que prenait le pousse-pousse. Celui-ci semblait entraîné par un poids énorme, comme si une force irrésistible l'eût attiré.

Chen ne s'en aperçut que trop tard./

(10) Quelle course il fit jusqu'au port! A grandes enjambées, il dévalait les ruelles en escalier, traversait les carrefours, effleurait à peine le sol. Derrière lui, ce n'était qu'étals renversés, passants jetés à terre et qui se relevaient en criant. Des gens le poursuivaient, lui lançaient des pierres et des injures.

- Ah le brigand! Le sacripant!

- Qu'on l'arrête! Qu'on le fouette !

Il entendait autour de lui un tonnerre qui grandissait, grandissait, et devenait assourdissant.

Il ne voyait que des obstacles qui accouraient et qu'il pulvérisait. /

(11) Et toujours, 
Toujours,

Le pousse-pousse volait vers la mer,

A tours de roues,

A coups de talons,

A orteils crispés.

Poitrine haletante,

Dans les rues descendantes,

Chen s'essoufflait.

Au bout de la dernière rue, un bassin du port s'étendait,

Calme,

profond,

sombre,

très sombre./

(12) Chen plongea comme en un rêve dans l'eau épaisse, les pieds en avant, et tout le poids du pousse-pousse infernal derrière lui./

Quand il revint à la surface, il vit que seul le chapeau et le pousse-pousse flottaient

De client, point.

Disparu.

Des matelots, au bord du bassin, riaient en se donnant de grandes claques sur les cuisses.

- Vous... vous n'avez pas vu un jeune homme ? demanda Chen.

- Où donc?

- Ben... dans mon pousse-pousse!

Ils rirent encore plus fort en l'accusant d'avoir bu trop d'alcool de riz.

- Ton pousse-pousse était vide !/

Pas de jeune homme? Que voulait donc dire cela?

Longtemps, Chen observa l'eau pour voir si son client remontait.

«Je n'ai pas rêvé pourtant! J’ai bien transporté un jeune homme ! »

Afin de chasser son inquiétude, il alla voir Wang dans sa cabane./

(13) Il trouva celui-ci tout pensif, le menton au creux de la main et le regard perdu au loin.

- Mon cormoran est mort, bredouilla Wang.

Chen en fut ému. Une pensée traversa son esprit, rapide, pointue, une de ces petites pensées qui ne vous laissent pas le choix et vous font dire :

- Si j'ai de l'argent ce soir, je te le donnerai./ 
(14) Le temps des mauvais calculs était bien fini. Noyés dans le port, les désirs d'égoïste en puissance, les rêves de richesse gagnés au prix de l'amitié.

Chen revint à son pousse-pousse. Il avait l'esprit libre de nouveau, le pied sûr au milieu de la foule. Le chapeau incliné sur l'oreille, il descendit dans la ville basse, sous les écriteaux multicolores./

(15) - Pst !

On l'appelait. Il s'arrêta. Un homme énorme occupait l'encadrement d'une porte. Chen considéra le menton qui faisait trois plis et le ventre qui en faisait quatre sous la robe richement brodée.

« Il va casser mon pousse-pousse !

Pourtant il s'approcha d'un pas hésitant.

— Où donc désire aller le Très Honorable Seigneur?

- Là-haut.

Chen interrompit le salut qu'il accordait, en s'inclinant, à ses clients d'importance. Il leva les yeux, puis le nez, puis se redressa complètement.

Là-haut!

Tout en haut de la colline qu'il habitait !

Il fallait tirer cet homme énorme tout en haut !

- Je sais... dit le mandarin avec un beau sourire.

Mais tu seras payé largement pour ta peine.

Chen pensa à la promesse qu'il avait faite à Wang.

- Bon! répondit-il en crachant dans ses mains./

(16) Le rotin gémit quand le noble personnage s'installa.

L'essieu fléchit, les roues donnaient l'impression d'être cloués au sol. Chen tendit ses jarrets maigres et, par un effort violent, arracha le pousse-pousse.

Il monta lentement, ruelle après ruelle.

La sueur lui brûlait les yeux. Les muscles de son cou étaient près de craquer tant le malheureux serrait les mâchoires pour se concentrer sur l'effort.

« Wang sera content », se répétait-il afin d'entretenir son courage./

(17) N'était-ce qu'une illusion ? Il lui parut, au bout d'un moment, que l'homme se faisait moins lourd, la côte moins abrupte.

Ce n'était pas une illusion. Chen, peu à peu, ne peina presque plus.

Cependant, il n'osait se retourner par crainte de perdre son élan et d'être emporté vers le bas.

- Arrête-toi, commanda la voix derrière lui. C'est ici.

Ils venaient d'arriver devant la maison aux kiosques de porcelaine./

(18) Chen se retourna et n'en crut pas ses yeux. 
Dans le pousse-pousse, à la place du mandarin, se trouvait le jeune homme du matin, souriant, avec sa natte brillante et sa robe couleur de soleil.

Il tendit à Chen une grosse pièce d'or et dit, sans cesser de sourire :

Ne t'étonne pas. Tu m'as vu jeune homme et puis mandarin. Tu me verras oiseau, peutêtre, ou rayon de lune... ou bâtonnet d'encens, que sais-je encore ? Je suis un génie. Je connais des humains toutes les bonnes pensées et toutes les mauvaises. Prends cet or. Tu l'as mérité. Et fais-en l'usage que tu as prémédité./

(19) Le génie s'évanouit en fumée. Une petite fumée bleue qui s'envola vers le portail pour se couler dans le trou de la serrure.

«Ai-je rêvé ? ", se demanda Chen.

Pourtant, l'or était bien réel au creux de sa main brune, et bien réelle aussi allait être la joie de Wang.

\section{NOTES}

1. Le terme valeur est défini plus loin.

2. Pour la notion de différenciation, nous renvoyons aux travaux de l'équipe Éducation, scolarisation (ESCOL) et, entre autres, à l'ouvrage d'E. Bautier et P. Rayou (2009).

3. En ligne : www.eduscol.education.fr, consulté le 10/04/14.

4. Parmi les extraits que l'on pourrait mettre en relation avec la description de la demeure du génie dans l'album que nous étudions, mentionnons, par exemple :

« [Le sultan] passa ensuite dans un salon merveilleux, au milieu duquel il y avait un grand bassin avec un lion d'or massif à chaque coin. Les quatre lions jetaient de l'eau par la gueule, et cette eau, en tombant, formait des diamants et des perles ; ce qui n'accompagnait pas mal un jet d'eau qui, s'élançant du milieu du bassin, allait presque frapper le fond d'un dôme peint à l'arabesque. Le château, de trois côtés, était environné d'un jardin que les parterres, les pièces d'eau et mille autres agréments concouraient à embellir ; et ce qui achevait de rendre ce lieu admirable, c'était une infinité d'oiseaux qui y remplissaient l'air de leurs chants harmonieux, et qui y faisaient toujours leur demeure » (Galland, $1965: 95-96)$.

5. L'état d'un sujet se définit par sa relation de jonction ou de disjonction avec un objet.

6. V. Jouve se réfère ici à la typologie de D. Cohn et note que le psycho-récit, le monologue narrativisé et le monologue rapporté favorisent par ordre décroissant l'investissement affectif du lecteur.

7. L'ouvrage que J.-L. Tilleuil (2005) a dirigé comporte une très complète bibliographie sur la relation texte-image, qui a été actualisée en 2007 (en ligne : http://grit.fltr.ucl.ac.be/IMG/pdf/ BiblioGrit02.pdf, consulté le 05/04/14).

8. Les Anglo-Saxons semblent plus prolixes sur la question de la relation texte/image dans les albums : par exemple, voir M. Nicolajeva et C. Scott (2000, 2001), F. Serafini (2010), M. Nicolajeva (2012) et L. R. Sipe (2012).

9. M. Joly (2011: 64) donne les définitions suivantes : « Au sein du message visuel, on distinguera les signes figuratifs ou iconiques, qui donnent de façon codée une impression de ressemblance avec la réalité en jouant sur l'analogie perceptive et sur les codes de représentation, héritées de la tradition représentative occidentale. Enfin on désignera sous le terme de signes plastiques les outils proprement plastiques de l'image tels que la couleur, les formes, la composition et la texture.» 
10. On trouve de ces dessins dans le manuscrit oriental Motifs illustrés des objets rituels de la cour impériale datant du XVIII ${ }^{\mathrm{e}}$ siècle.

11. Sur chaque double page ou presque (comme dans la plupart des pages de l'album), figurent des caractères chinois, dont le lecteur ne sait pas s'ils sont le fait de l'auteur, de l'illustratrice ou le fruit de leur concertation. Un petit dictionnaire est donné au lecteur, dans les premières pages, pour lui permettre de les lire (ils sont classés dans ce dictionnaire par ordre d'apparition).

\section{RÉSUMÉS}

À partir de l'étude d'un exemple en littérature de jeunesse fictionnelle, cet article s'intéresse au rôle que joue la relation texte/image dans l'orientation, en termes de valeurs, des supports pluricodés que constituent les albums.

Après une approche générale du texte de l'album Le Génie du pousse-pousse qui met l'accent sur l'orientation axiologique de ce texte, l'étude porte sur trois doubles-pages. Elle montre d'abord la complémentarité du texte et de l'image dans la construction de l'univers mis en place par le récit. Puis, elle met en évidence le fait que l'image, selon les pages, oriente l'album, du point de vue des valeurs, de trois manières : en revêtant une dimension proleptique anticipatrice du dénouement, en renforçant les jeux d'oppositions présents dans le texte, enfin, en montrant ce qui peut être plus ou moins inféré des pensées de personnages et que le texte ne dit pas.

Through the analysis of an example from children's literature, this article is interested in the role of the relationship between text and images and its influence on values in visual-verbal narrative books.

After a general approach of the text of the picturebook Le Génie du pousse-pousse, approach which emphasizes the axiological orientation of the text, the study focuses on three double pages. It shows at first the complementarity of text and image in the construction of the universe set up by the narrative. Then it reveals the fact that images, according to pages, orientate the picturebook, from the point of view of values, in three different ways : they adopt a proleptical dimension foretelling the resolution of the narrative, they strengthen the interplay of oppositions in the text, and they show what can be inferred on characters' thoughts without the need of clues in the text.

\section{INDEX}

Keywords : children's literature, picturebook, value, semiotics, image-text relationship

Mots-clés : albums, valeurs, sémiotique, relation texte/image

\section{AUTEUR}

\section{ANNE LECLAIRE-HALTÉ}

Crem (EA 3476), université de Lorraine (Espé) 